\title{
AN ADAPTIVE MULTISCALE METHOD FOR SIMULATION OF FLUID FLOW IN HETEROGENEOUS POROUS MEDIA
}

\author{
JØRG E. AARNES \& YALCHIN EFENDIEV*
}

\begin{abstract}
Several multiscale methods for elliptic problems that provide high resolution velocity fields at low computational cost have been applied to porous media flow problems. However, to achieve enhanced accuracy in the flow simulation, the numerical scheme for modeling the transport must account for the fine scale structures in the velocity field. To solve the transport equation on the fine scale with conventional finite volume methods will often be prohibitively computational expensive for routine simulations. In this paper we propose a more efficient adaptive multiscale method for solving the transport equation. In this method the global flow is computed on a coarse grid scale, while at the same time honoring the fine scale information in the velocity field. The method is tested on both two- and three-dimensional test cases with complex heterogeneous structures. The numerical results demonstrate that the adaptive multiscale method gives nearly the same flow characteristics as simulations where the transport equation is solved on the scale of an underlying fine grid. Some analysis is presented to estimate error sources and support our conclusions from the numerical results.
\end{abstract}

Key words. multiscale methods, mixed finite element methods, porous media, pseudofunctions, upscaling

AMS subject classifications. 65N99, 76T99, 76S05, 35L99

1. Introduction. Modeling flow in porous media is important in many areas of science. Unfortunately, the high degree of variability, uncertainties and multiscale nature of formation properties such as permeability pose significant challenges for subsurface flow modeling. Geological characterizations that capture these effects are typically developed at scales that are too fine for direct flow simulation because of limited computer power and memory. Simulations are therefore usually performed on coarsened models with a significantly reduced set of parameters.

As an alternative to performing flow simulations on coarsened models, the use of multiscale methods to do flow simulations directly on geostatistical models have been proposed. In this paper we explore one such option for modeling two-phase flow in porous media. The proposed methodology is based on a two-phase flow model consisting of an elliptic (or parabolic) equation for pressure and velocities, and a hyperbolic (or parabolic) equation that models the phase transport. The main ingredients in this model is Darcy's law that expresses flow velocities as functions of the pressure gradient and the pull down force due to gravity, combined with the continuity, or mass balance equations for each phase.

Multiscale methods for solving elliptic equations have been proposed by many authors, e.g., variational multiscale methods $[5,6]$, multiscale finite element methods $[7,10,20,1]$, multiscale finite volume method [21], equation-free computation [22], and methods based on the heterogeneous multiscale method (HMM) framework (e.g., [15]). In this paper we will use a variant of the method proposed in [10] that, in addition to giving mass conservative velocity fields on the discretization grid (the coarse grid), provides a mass conservative velocity field on an underlying subgrid. This feature allows users to choose grids for flow transport simulations in a nearly seamless fashion.

* The first author is partially supported by the Research Council of Norway under grant no. 158908/I30. The research of the second author is partially supported by NSF grants DMS-0327713 and DOE grant DE-FG02-05ER25669. 
By using multiscale methods to produce conservative velocity fields on fine grids, it is in principle possible to simulate the flow transport on high resolution geological models. But, to model transport on very large geological models requires more efficient simulation techniques than commercial simulators can offer today. The simulators that can handle the largest models, with a capacity of $10^{6}-10^{7}$ grid cells, are based on using streamline methods to model the flow transport, and a standard method to solve the pressure equation. Thus, a seemingly appealing alternative, that was explored in $[3,19]$, is to use streamline methods for the flow transport together with a multiscale method for solving the pressure equation. However, the use of streamline methods is still disputed, partly because they usually neglect effects from capillary forces, but also because modeling compressibility with streamline methods is complicated, and requires quite a bit of bookkeeping (see e.g., [27]). Thus, although streamline techniques have demonstrated their value in many applications, finite volume methods still dominate in reservoir and groundwater flow simulation. But if finite volume methods are to be used to compute flow transport on multi-million cell geological models, then remedies that prevent excessive computational cost must be employed.

The main objective in this paper is to propose an adaptive multiscale method for solving transport equations that arise in two-phase flow models. The method is based on a finite volume methodology and resolves both coarse scale and fine scale flow patterns. In this paper we neglect capillary forces so that the transport equation is hyperbolic. To develop a multiscale method for hyperbolic equations describing twophase flow dynamics, where the velocity field is heterogeneous and varies in time, is challenging. For nearly time-independent velocity fields, it can be advantageous to do upscaling and/or develop multiscale methods using a streamline coordinate system $[26,27]$. Indeed, upscaling along the streamline coordinate, i.e., in the direction of the flow, is almost trivial. However, upscaling across the streamlines is difficult because non-local terms can appear. Thus, although streamline coordinate systems have advantages in upscaling of two-phase flows [26], they allow only moderate changes in the velocity field, and are mostly well suited for convection dominated equations.

In general, there is a strong need for multiscale methods in Cartesian coordinate systems, such as the one proposed in this paper, which are not limited to convection dominated equations and can handle various physical processes without significant modifications. The key idea of the proposed multiscale method is to use information from a velocity field with subgrid resolution to improve accuracy of flow simulations on coarse grids. This is accomplished by introducing an operator that, at each timestep, maps the coarse grid saturation profile onto a plausible saturation solution on the underlying subgrid. To improve accuracy of the subgrid solution, one can adaptively replace the multiscale solution in regions with sharp fronts with a solution of a localized version of the transport equation.

Finally we remark that the proposed adaptive multiscale method has some similarities with the multiscale framework developed for nonlinear equations [18], and with pseudo type approaches $[23,8,25]$. But important differences between these methods and our approach do exist, and these will be discussed in the paper.

The paper is organized as follows. In Section 2 we introduce equations for immiscible and incompressible two-phase flow. In Section 3 we present the multiscale mixed finite element method [4] that is used to compute flow velocities. In Section 4 we describe the new multiscale method for solving the transport equation. In Section 5 we discuss the relation between the proposed multiscale method and the more traditional upscaling approaches for the saturation equation based on using pseudo relative 
permeabilities. The general analysis is presented in Section 6. In Section 7 we present some numerical results that demonstrate the performance of the proposed adaptive multiscale method for hyperbolic transport equations in two-phase flow models.

2. Mathematical model. We consider a model problem for immiscible and incompressible two-phase flow where we neglect effects from gravity and capillary pressure. The equations are derived from conservation of mass for each phase $j$ :

$$
\phi \frac{\partial S_{j}}{\partial t}+\nabla \cdot v_{j}=q_{j}
$$

where the phase velocities $v_{j}$ are given by Darcy's law:

$$
v_{j}=-\lambda_{j}\left(S_{j}\right) K \nabla p_{j} .
$$

Here $\phi$ is the porosity, $S_{j}$ is the $j$-phase saturation (fraction of the void occupied by phase $j$ ) and $q_{j}$ is a source (or sink) term. In Darcy's law, $K$ is the permeability tensor, $p_{j}$ is the phase pressure and $\lambda_{j}$ is the phase mobility given by $\lambda_{j}\left(S_{j}\right)=k_{r j}\left(S_{j}\right) / \mu_{j}$, where $k_{r j}$ and $\mu_{j}$ are the relative permeability and viscosity of phase $j$ respectively. The relative permeability is function of the saturations only, and models the reduced conductivity of a phase due to the presence of other phases.

Let the two phases be oil and water $(j=o, w)$. Since we neglect effects from capillary pressure so that $\nabla p_{o}=\nabla p_{w}$, we assume $p_{o}=p_{w}=p$. Then the Darcy equations combined with conservation of mass yields the pressure equation:

$$
\begin{aligned}
v & =-\left(\lambda_{w}+\lambda_{o}\right) K \nabla p, \\
\nabla \cdot v & =q,
\end{aligned}
$$

where $v=v_{w}+v_{o}$ and $q=q_{w}+q_{o}$. If we assume that the two phases occupy the void space completely, and introduce the water fractional flow $f_{w}=\lambda_{w} / \lambda$ where $\lambda\left(S_{w}\right)=\lambda_{w}\left(S_{w}\right)+\lambda_{o}\left(1-S_{w}\right)$ is the total mobility, then we may write the conservation equation for water, henceforth called the saturation equation, as follows:

$$
\phi \frac{\partial S_{w}}{\partial t}+\nabla \cdot\left(f_{w} v\right)=q_{w}
$$

In the following we will, for ease of notation, drop the $w$-subscripts of $S_{w}$. As in [1], the system of equations (2.3)-(2.4) will be solved using a sequential splitting, where the pressure equation is solved at the current time-step using saturation values from the previous time-step.

3. A mixed multiscale FEM for elliptic problems. Let $\Omega \subset \mathcal{R}^{d}$, and denote by $n$ the outward pointing unit normal on $\partial \Omega$. In mixed formulations of $(2.3)$ with no-flow boundary conditions on $\partial \Omega$, one seeks $(v, p) \in H_{0}^{1, \text { div }}(\Omega) \times L^{2}(\Omega)$ such that

$$
\begin{aligned}
& \int_{\Omega} v \cdot(\lambda K)^{-1} u d x-\int_{\Omega} p \nabla \cdot u d x=0 \quad \text { for all } u \in H_{0}^{1, \operatorname{div}}(\Omega), \\
& \int_{\Omega} l \nabla \cdot v d x=\int_{\Omega} q l d x \quad \text { for all } l \in L^{2}(\Omega) .
\end{aligned}
$$

Here $H_{0}^{1, \operatorname{div}}(\Omega)=\left\{v \in\left(L^{2}(\Omega)\right)^{d}: \nabla \cdot v \in L^{2}(\Omega)\right.$ and $v \cdot n=0$ on $\left.\partial \Omega\right\}$. Note that to determine $p$, one must add an additional constraint such as $\int_{\Omega} p=0$. 
In a mixed FEM discretization of (3.1)-(3.2), the spaces $H_{0}^{1, \text { div }}(\Omega)$ and $L^{2}(\Omega)$ in which we seek the pressure and velocity solutions are replaced by finite dimensional subspaces, say $U$ and $V$, that typically consists of low order piecewise polynomials. In a MsMFEM one attempts to design the approximation space for velocity in such a way that it embodies the impact of subgrid variations in $K$. The MsMFEM outlined below [4] is a variant of the method introduced by Chen and Hou [10] that, in addition to giving mass conservative velocity fields on the discretization grid (the coarse grid), provides a mass conservative velocity field on an underlying subgrid. To formulate the method in [4], let $\mathcal{T}=\left\{T_{i}\right\}$ be a grid where each grid block is a connected union of cells in an underlying subgrid $\mathcal{K}=\left\{K_{i}\right\}$. The grid $\mathcal{T}$ will be referred to as the coarse grid, and the subgrid $\mathcal{K}$ will be referred to as the fine grid. Let the approximation space for pressure be the space of piecewise constant functions on $\mathcal{T}$, i.e.,

$$
U=\operatorname{span}\left\{u \in L^{2}(\Omega):\left.u\right|_{T} \text { is constant for all } T \in \mathcal{T}\right\} .
$$

To define the approximation space $V$ for velocity $v$, denote by $\Gamma_{i j}=\partial T_{i} \cap \partial T_{j}$ the nondegenerate interfaces in the coarse grid. To each interface, we assign a corresponding basis function $\psi_{i j}$. This basis function is supported in $\Omega_{i j}=T_{i} \cup \Gamma_{i j} \cup T_{j}$, and is related to a function $\phi_{i j}$ through Darcy's law: $\psi_{i j}=-\lambda K \nabla \phi_{i j}$. The function $\phi_{i j}$ is obtained by solving (numerically) the following local elliptic problem:

$$
\psi_{i j} \cdot n_{i j}=0 \text { on } \partial \Omega_{i j}, \quad \nabla \cdot \psi_{i j}=\left\{\begin{array}{cl}
f_{i}(x) / \int_{T_{i}} f_{i}(x) d x & \text { for } x \in T_{i}, \\
-f_{j}(x) / \int_{T_{j}} f_{j}(x) d x & \text { for } x \in T_{j} .
\end{array}\right.
$$

Here $n_{i j}$ is the outward pointing unit normal to $\partial \Omega_{i j}$, and

$$
f_{i}=\left\{\begin{array}{l}
f \text { if } \int_{T_{i}} f d x \neq 0 \\
\lambda \operatorname{trace}(K) \text { else }
\end{array}\right.
$$

The MsMFEM approximation space for velocity is now the span of the basis functions $\left\{\psi_{i j}\right\}$. The source terms $\left\{f_{i}\right\}$ are chosen as defined by (3.4) for the following reasons. First, they produce basis functions that force unit flux across associated coarse grid interfaces, i.e., $\int_{\Gamma_{i j}} \psi_{i j} \cdot n d s=1$, where $n$ is the unit normal to $\Gamma_{i j}$ pointing into $T_{j}$. This implies that the MsMFEM solution $\left\{v_{i j}\right\}$ for velocity gives the fluxes across the respective coarse grid interfaces. Second, if a conservative method is used to compute basis functions, then the velocity $v=\sum v_{i j} \psi_{i j}$ conserves mass on the subgrid $\mathcal{K}$. Third, the special source terms in blocks with a source allows the method to model radial flow around point or line sources, such as wells in oil-reservoirs, on the subgrid scale. Finally, by letting $f_{i}$ scale according to the permeability as in (3.4), one can to some extent avoid unnaturally high velocities in low-permeable fine grid cells. We would like to note that global information can be used to achieve high accuracy in MsMFEM, as it was demonstrated in [1] (cf. [17]).

The computational complexity of a MsMFEM is not significantly less than the computational complexity of solving the full problem on the fine grid with a (very) efficient linear solver. Note, however, that the most expensive task in a MsMFEM, the computation of the basis functions is a so-called embarrassingly parallel computation since each basis function $\psi_{i j}$ can be computed independently. Another advantage for the purpose of running two-phase flow simulations, for which the pressure equation (2.3) needs to be solved multiple times due to dynamic changes in the mobility $\lambda$, is that basis functions often need to be recomputed only when flow conditions change 
significantly. This claim is supported by an analysis of the saturation dependence in the pressure solution [17]. Here it was shown that when flow conditions do not change, the time varying velocity field is strongly influenced by the initial velocity field. The computation of basis functions then becomes part of a preprocessing step, and MsMFEMs become analogous to single-phase flow upscaling methods, see e.g., [11] and references therein. The computational complexity of a MsMFEM is also comparable to the computation cost of single-phase flow upscaling procedures. We should add, however, that if saturation profiles exhibit sharp fronts, then a slight improvement in accuracy can be obtained by regenerating basis functions in regions where the saturation has changed substantially since the previous time-step [1]. Similar observations were made by Jenny et al. [21] for the multiscale finite volume method.

4. A multiscale method for hyperbolic equations. The adaptive multiscale method that we propose here consists of two parts. An adaptive criteria determines if a block is in a transient flow region. Here, transient region refers to the regions with sharp saturation fronts. In these regions we use local fine grid computations to advance the saturation solution to the next time-step. In regions with slow transients, we use a multiscale coarse grid solver to advance the saturation solution to the next time-step. Then, instead of doing a fine-grid calculation, we map the coarse grid solution onto a fine grid solution using special interpolation operators.

Before we give an outline of the algorithm, we need to introduce some additional notation. First, denote, as in Section 3, the coarse grid by $\mathcal{T}=\left\{T_{i}\right\}$ and an underlying fine grid by $\mathcal{K}=\left\{K_{i}\right\}$. Although we employ the same notation as in Section 3, the grids used here need not coincide with the coarse and fine grids for MsMFEM and can be unstructured. As for MsMFEM, the only restriction on the coarse grid is that each grid block consists of a connected union of grid cells in the fine grid. Moreover, the only constraint on the fine grid is that each grid cell consists of a collection of grid cells in the fine grid for MsMFEM. This constraint ensures that MsMFEM provides fluxes across all fine grid interfaces $\left\{\gamma_{i j}=\partial K_{i} \cap \partial K_{j}\right\}$.

We introduce now the upstream fractional flow function for $\gamma_{i j}$ :

$$
V_{i j}(S)=\max \left\{v_{i j} f_{w}\left(S_{i}\right),-v_{i j} f_{w}\left(S_{j}\right)\right\},
$$

where $v_{i j}$ is the Darcy flux across $\gamma_{i j}$ that we get from the MsMFEM solution. Next, let $\bar{S}_{i}^{n}$ be the coarse grid saturation in $T_{i}$ at time $t_{n}$, and denote by $\mathcal{T}_{\mathrm{tr}}^{n}$ the family of grid blocks that are identified to be in a transient flow region. One can use various criteria based on coarse-scale saturation values or its gradients to identify transient regions. In this paper, the following criterion is used to identify transient flow regions:

$$
T_{i} \in \mathcal{T}_{\mathrm{tr}}^{n} \text { if } \max \left\{\left|\bar{S}_{i}^{n}-\bar{S}_{j}^{n}\right|:\left|\partial T_{i} \cap \partial T_{j}\right|>0\right\} \geq \alpha_{i} .
$$

For each $T_{i} \in \mathcal{T}_{\text {tr }}^{n}$, we define

$$
T_{i}^{E}=T_{i} \cup\left\{K \in \mathcal{K}:\left|\partial K \cap \partial T_{i}\right|>0\right\} .
$$

Hence, $T_{i}^{E}$ consist of grid cells that are either contained in $T_{i}$, or that share a common interface with a cell in $T_{i}$. Finally, we introduce a family of operators $\left\{I_{T}: T \in \mathcal{T}\right\}$ that map coarse grid saturations onto fine grid saturation fields inside the respective blocks. The adaptive multiscale method is now outlined in Algorithm 4.1.

Note that in the adaptive multiscale method outlined in Algorithm 4.1, we update first fine-grid saturations in transient flow regions, and then coarse grid saturations in 
non-transient regions using (4.3). Finally we map the coarse grid saturations in nontransient regions onto the fine grid with the coarse-to-fine grid interpolation operators. There is no constraints on the time-steps $\triangle t$, but they should be chosen small enough to avoid excessive numerical diffusion. However, since (4.3) is an explicit scheme, the time-steps $\triangle_{j} t$ are constrained by a CFL-condition. The time-step $\triangle_{j}$ is given by

$$
\triangle_{j}=\min \left\{\triangle t-\sum_{i=0}^{j-1} \triangle_{i} t, \frac{\delta}{2}\right\}
$$

where $\triangle_{0}=0$ and $\delta$ is determined by the following condition for stability:

$$
0 \leq \bar{S}_{T}^{n+1}+\frac{\delta}{\int_{T} \phi d x}\left[\int_{T} q_{w}\left(S^{n+1}\right) d x-\sum_{\gamma_{i j} \subset \partial T} V_{i j}\left(S^{n+1}\right)\right] \leq 1 .
$$

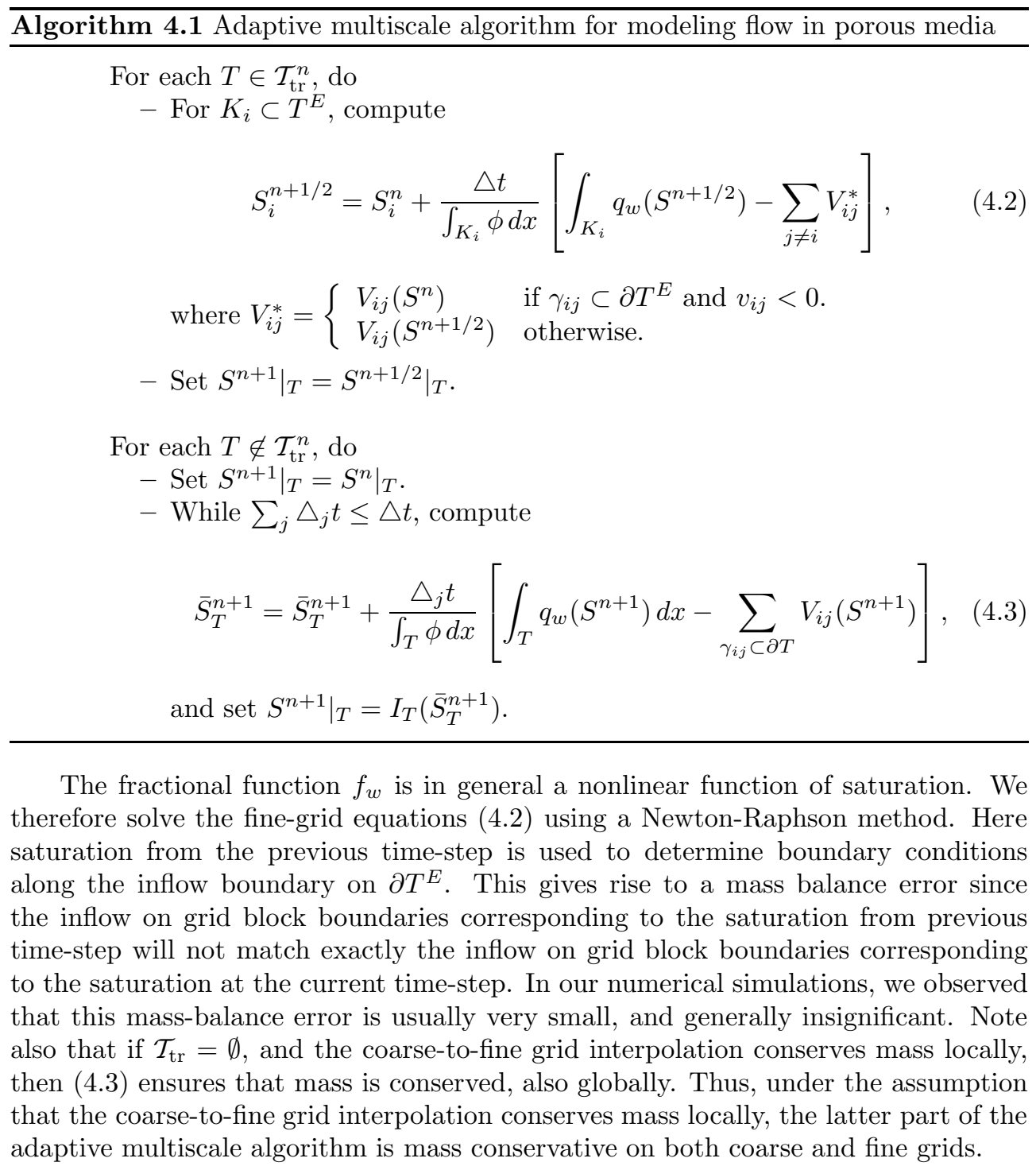


Next, observe that fluxes across coarse grid interfaces in (4.3) are evaluated on fine grid interfaces $\gamma_{i j} \subset \partial T$. Thus, rather than using a flux function that models the total flux across coarse grid interfaces as a function of the net saturation in the upstream block, we evaluate the term $f_{w} v$ in (2.4) on the scale of the fine grid. This requires that we have fine grid saturation values in all cells adjacent to grid block boundaries. The coarse-to-fine grid interpolation operators $\left\{I_{T}\right\}$ are therefore not just tools to get better resolution. In addition to improving the global accuracy of Algorithm 4.1 by providing a better approximation to flow across coarse grid interfaces, they provide initial fine grid saturation values for (4.2) in the transition when a block is identified as being part of a transient flow region. Without the interpolation, the initial saturation field for (4.2) would be constant in $T$, and the fractional flow across the coarse grid interfaces would have to be based on the net grid block saturations only, as pseudofunctions generally do $[23,25,8]$.

We remark that the proposed adaptive multiscale method has some similarities to the multiscale framework developed for nonlinear equations [18] in which multiscale basis functions are constructed by mapping the coarse dimensional space defined over the entire region. Furthermore, this map is used in the global coarse-grid formulation of the fine-scale problem to compute the coarse-scale solution. In our multiscale approach, the basis functions are constructed as a function of average saturation in each coarse block, and then used in the global formulation of the problem. In both approaches, the main task is to determine an accurate and efficient multiscale map that improves the global coarse-grid formulation of the problem.

4.1. The coarse-to-fine grid interpolation operator. In the following we attempt to construct operators that map each coarse grid saturation field onto a fine scale saturation profile that is close to the corresponding profile that one would get by solving saturation equation on the global fine grid. The basic idea is to approximate the fine grid saturation in $T_{i}$ as a linear combination two basis functions $\Phi_{i}^{k}$ and $\Phi_{i}^{k+1}$ with $\int_{T_{i}} \Phi_{i}^{k} \phi d x \leq \bar{S}_{i}^{n} \int_{T_{i}} \phi d x<\int_{T_{i}} \Phi_{i}^{k+1} \phi d x$ :

$$
I_{T_{i}}\left(\bar{S}_{i}^{n}\right)=\omega \Phi_{i}^{k}+(1-\omega) \Phi_{i}^{k+1} .
$$

Here $\omega \in[0,1]$ is chosen such that the interpolation preserves mass, i.e., such that

$$
\int_{T_{i}} I_{T_{i}}\left(\bar{S}_{i}^{n}\right) \phi d x=\bar{S}_{i}^{n} \int_{T_{i}} \phi d x .
$$

This condition states that the fluid contained in $T_{i}$ is distributed inside $T_{i}$ in such a way that the total fluid volume in $T_{i}$ is conserved. The basis functions $\Phi_{i}^{k}=\chi_{i}\left(x, \tau_{k}\right)$ represent snapshots of the solution of the following equation:

$$
\phi \frac{\partial \chi_{i}}{\partial t}+\nabla \cdot\left(f_{w}\left(\chi_{i}\right) v\right)=q_{w} \quad \text { in } T_{i} .
$$

For the local problem (4.6) to be well-defined, we need to specify initial conditions and boundary conditions, and provide a possibly time-varying velocity field in $T_{i}$. Unfortunately, we do not know a priori what the velocity will be during the simulation, nor what boundary conditions to impose. Assumptions must therefore be made to approximate how the velocity and saturation evolves. We describe below an approach which is local in terms of boundary and initial conditions, however, one can naturally incorporate global information into this approach. The proposed approach assumes that global boundary conditions for the pressure equation (2.3) are not changed, and 
that the source term $q$ is fixed. We assume also that an upstream method is used to solve the local equations (4.6). Thus, we need only specify boundary conditions on the inflow boundaries $\Gamma_{T}^{\text {in }}=\left\{\gamma_{j l} \subset \partial T: K_{l} \subset T, v_{j l}<0\right\}$.

For fixed flow conditions, the fine scale velocity features will generally not change significantly during a flow simulation. This is discussed in Section 6. One option is therefore to solve the pressure equation (2.3) at initial time with MsMFEM, use $v=\left.v\left(x, t_{0}\right)\right|_{T}$ in (4.6), and the same initial data as for the global problem (2.4). A local way of generating saturation basis functions based on this approach requires that sensible boundary conditions for (4.6) can be imposed for each block independently.

In this paper, we impose $f_{w}=1$ on the inflow boundary $\Gamma_{T}^{\text {in }}$. For general coarse grids, these boundary conditions may seem a bit crude. Indeed, these boundary conditions are exact only if there is a sharp front in the global solution that, for each block, hits the whole inflow boundary at approximately the same instant. It should be emphasized that the purpose of the interpolators is not primarily to get the fine scale details correct, but rather to introduce a flexible mechanism that allows us to model the flow on a coarse scale more correctly.

To get accurate solutions, also on fine grids, one must either use an adaptive component to improve the solution in transient flow regions, or build more information into the interpolators. For instance, note that the inherent flexibility with respect to coarse grids allows us to reduce the error associated with this type of boundary conditions by using flow-based, non-Cartesian grids. In particular, by using coarse blocks with boundaries aligned with level sets of time-of-flight function (see Section 6 for definition), one can achieve higher accuracy compared to the approaches where Cartesian coarse blocks (or coarse blocks selected independent of global flow features) are used. This option is discussed further in Section 6. We note, however, that our numerical results in Section 7 show that the multiscale approach using Cartesian coarse blocks still provide a good overall accuracy.

In summary, the interpolation operators $I_{T}$ are computed using a fixed velocity field, and static boundary conditions on $\partial T$. One can use a limited global information, such as time-of-flight function, in constructing coarse blocks. The use of Cartesian coarse blocks (or coarse blocks selected independent of global flow features) also provides a good overall accuracy despite the fact that the history dependence is neglected by not taking into account travel times from injectors to the inflow boundary. However, since the interpolation mapping is used only in regions with moderate transient flow, neglecting the history dependence has limited impact on the global flow behavior, as we observe in our numerical simulations.

5. The relation to pseudo type approaches. An approach that is often used in practice for upscaling the saturation equation entails the use of so-called pseudo relative permeabilities $\left(k_{r j}^{*}\right)_{i}=\left(k_{r j}^{*}\right)_{T_{i}}$ in place of the fine scale $k_{r j}$. Because the fine scale $k_{r j}$ are typically functions only of saturation $S$, pseudo relative permeabilities, or pseudofunctions for brevity, are commonly assumed to depend only on the coarse grid saturation $\bar{S}$, though the curves can vary between coarse grid blocks. Note that we use the overbar to designate coarse grid velocity and saturation (which can be thought of as volume averaged fine grid quantities, where the volume average is over the region corresponding to the coarse grid block). With this representation for the pseudofunctions, the coarse scale saturation equation in the absence of gravity forces and capillary pressure effects becomes:

$$
\frac{\partial \bar{S}}{\partial t}+\nabla \cdot F^{*}(x, \bar{S})=0,
$$


where $F^{*}(x, \bar{S})=\bar{v} f_{w}^{*}, \bar{v}$ is the upscaled velocity field. Here the variation of $F_{i}^{*}$ with $x$ appears because the pseudofunctions are in general different for each block in the coarse grid. The upscaled flux function $f_{w}^{*}=\left\{f_{w, i}^{*}\right\}$ is defined via the usual relation between the flux function and the relative permeabilities:

$$
f_{w, i}^{*}=\frac{\left(k_{r w}^{*}\right)_{i} / \mu_{w}}{\left(k_{r w}^{*}\right)_{i} / \mu_{w}+\left(k_{r o}^{*}\right)_{i} / \mu_{o}} .
$$

The pseudofunctions are computed from local fine scale problems such that they provide the same average response as the fine grid model for the prescribed boundary conditions. Assuming that the pseudofunctions have been computed, the corresponding coarse scale equation takes the following form:

$$
\bar{S}^{n+1}=\bar{S}^{n}+\frac{\Delta t}{\int_{T} \phi d x}\left[\int_{T} q_{w}\left(S^{n}\right) d x-\sum_{\Gamma_{i j} \subset \partial T} V_{i j}^{*}\left(S^{n}\right)\right],
$$

where $V_{i j}^{*}(S)=\max \left\{\bar{v}_{i j} f_{w, i}^{*}\left(\bar{S}_{i}\right),-\bar{v}_{i j} f_{w, j}^{*}\left(\bar{S}_{j}\right)\right\}$. Note that (5.3) is similar to (4.3). The main difference is that in (4.3) we employ the original fractional flow function $f_{w}$ and sum over the fine grid interfaces to evaluate $\int_{\partial T} f_{w} v \cdot n d s$.

Apart from the fact that the coarse scale equations are similar, the construction of the interpolation operator in the proposed multiscale approach has some similarities with the construction of the pseudofunctions if Cartesian coarse blocks are used and $S=1$ is imposed at the inflow boundaries. Indeed, pseudofunctions are generated by solving a set of local flow problems that resemble (4.6) for each grid block. Thus, both approaches have to address the problem of finding appropriate boundary conditions on the inflow boundaries. Moreover, the computational complexity of computing the saturation basis functions is comparable to the corresponding complexity of generating pseudofunctions. There are, however, important differences between pseudofunction based flow simulation and the proposed multiscale approach, both in terms of how subgrid effects are modeled on coarse grids, and in the way that the pseudofunctions and interpolation operators are generated respectively. We elaborate now on the latter part and discuss how the procedure for generating pseudofunctions differ from the generation of the interpolation operators through (4.6).

In (4.6) we employ a velocity field obtained by solving the saturation equation at initial time on the global fine grid. In contrast, a velocity field corresponding to a local two-phase flow system is used to generate pseudofunctions. This means that in addition to saturation boundary conditions on inflow boundaries, one has to specify artificial boundary conditions for a local pressure equation to create a flow through the grid block. Hence, whereas the multiscale approach neglects the dynamic nature of the velocity field by using a fixed velocity field, pseudo type approaches ignore effects of global boundary conditions. In Section 6.2 we justify our approach by showing that the dynamic two-phase flow velocity is strongly influenced by the initial velocity field under some assumptions. In particular, we argue that the local saturation solutions that we get from (4.6) using the initial velocity field match closely the local saturation solutions that one would get from (4.6) if the dynamic two-phase flow velocity field is used. For pseudo type approaches, the fact that artificial boundary conditions are used to force the flow implies that there is no apparent correspondence between the velocity fields used to generate pseudofunctions, and the two-phase flow velocity fields on the global fine-grid that correspond to the true boundary conditions. 
In principle one could use the initial global fine scale velocity, also to generate pseudo functions. However, pseudofunctions are designed to capture the average flow response on coarse grids given some underlying flow model. To this end one has to account for different flow scenarios where the moving front approaches the grid block from different angles. For instance, when generating pseudofunctions for each interface in the simulation grid, it is customary to define two pseudofunctions for each interface to account for flow in opposite directions. The proposed multiscale technique avoids this complexity. The multiscale technique also allows more resolution in the sense that it handles cases with cross-flow, i.e. flow in opposite directions across interfaces, in a manner that is more consistent with the fine grid model. In the upscaled model (5.3) one allows only one flow direction for each coarse grid interface.

Another problem with pseudo type approaches is that they can lead to a lack of robustness, in part because the upscaled form (5.1) for the coarse scale saturation equation is incomplete. Specifically, it has been shown previously [14] that the correct form of the upscaled saturation equation is more general than (5.1) in that it contains averages of products of subgrid (fluctuating) quantities such as $\overline{v^{\prime} S^{\prime}}$, where' designates a fluctuating quantity. These extra terms could be represented, for systems of large correlation length, as length and time dependent dispersivities [16]. In general, these terms need to be modeled, which requires often restrictive assumptions. In contrast, the multiscale approach presented in the paper do not require the form of the upscaled equations and allow to compute quantities such as $\overline{v^{\prime} S^{\prime}}$ on-the-fly. There are a number of advantages associated with the computations on-the-fly. First, it allows to perform down-scaling in the regions of interest which makes the method adaptive. Second, it would allow us to model the coarse-scale quantities, as a function of $\overline{v^{\prime} S^{\prime}}$, and avoid the closure assumptions involved in modeling this type of terms.

6. Analysis. In this section we discuss the quality of saturation equation solutions obtained using the adaptive multiscale algorithm. The analysis holds away from the sharp fronts. Recall that near sharp fronts, where the analysis breaks down, the transport equation is solved on the fine grid. Moreover, the coarse grid is unstructured, in general, and can be chosen using some limited global information. We would like to note that the analysis presented below is formal, though can be justified with mathematical rigor.

6.1. Direct estimates and error sources. First, we attempt to understand the error sources and provide some direct estimates. To this end, let

$$
\bar{S}^{n}=\frac{1}{|T|} \int_{T} S^{n} d x
$$

where $S^{n}=S\left(x, t_{n}\right)$ is the fine scale saturation field at time $t=t_{n}$, and denote by $S_{h}^{n}$ the corresponding saturation field obtained using the proposed multiscale technique.

We assume henceforth that the velocity field used in (4.6) is exact, i.e. that $v=v\left(x, S\left(x, t_{0}\right)\right)$ at all times $t>0$, and neglect the source terms. In general, there are errors in the velocity field due to the fact that the single-phase flow velocity is used in the computation of the interpolation operators. However, as we will show in Section 6.2 , the velocity used in the local problems is accurate under some assumptions in the sense that it provides approximately the same saturation field as if the actual two-phase flow velocity is used in the local problems.

Since we now disregard source terms and errors in the velocity, it makes sense to 
define auxiliary functions that controls the mass balance. Thus, let

$$
\begin{aligned}
G_{f}(S) & =-\frac{1}{\int_{T} \phi d x} \int_{\partial T} f_{w}(S)(v \cdot n) d s \\
G_{c}(\bar{S}) & =-\frac{1}{\int_{T} \phi d x} \int_{\partial T} f_{w}(I(\bar{S}))(v \cdot n) d s .
\end{aligned}
$$

Here $n$ is the outward unit normal on $\partial T$ and $I=I_{T}$ is the interpolation operator defined in Section 4.1. Note that since $v$ is fixed, the saturation field $S(x, t)$ is a monotonically increasing function of time. In other words, if $S\left(\cdot, t_{1}\right)=S\left(\cdot, t_{2}\right)$ then $t_{1}=t_{2}$. Thus, if $S_{2} \geq S_{1}$ for some $x \in T$, then $S_{2} \geq S_{1}$ for all $x \in T$. Similarly the interpolation $I(\bar{S})$ produces saturation fields that increase monotonically with $\bar{S}$. As a direct consequence, we have that $G_{f}$ and $G_{c}$ increase monotonically in the sense that if $S_{2}>S_{1}$ for some $x \in T$, then $G_{f}\left(S_{2}\right) \geq G_{f}\left(S_{1}\right)$ and $G_{c}\left(\bar{S}_{2}\right) \geq G_{c}\left(\bar{S}_{1}\right)$.

Observe now that with the notations above, we have

$$
\begin{aligned}
& \bar{S}^{n+1}-\bar{S}^{n}=G_{f}\left(S^{n+1}\right) \Delta t+o(\Delta t), \\
& \bar{S}_{h}^{n+1}-\bar{S}_{h}^{n}=G_{c}\left(\bar{S}_{h}^{n+1}\right) \Delta t .
\end{aligned}
$$

For simplicity, the remainder is denoted by $o(\Delta t)$. Note that this error is due to temporal discretization (backward Euler). If the average saturation is a smooth function with respect to time then the remainder is $O\left((\Delta t)^{2}\right)$.

We are primarily interested in how much $S_{h}^{n}$ deviates from $S^{n}$. Hence, let

$$
\delta^{n}=\bar{S}^{n}-\bar{S}_{h}^{n} .
$$

Subtracting the equations (6.1) and (6.2) now gives

$$
\begin{array}{r}
\delta^{n+1}-\delta^{n}=G_{f}\left(S^{n+1}\right) \Delta t-G_{c}\left(\bar{S}_{h}^{n+1}\right) \Delta t+o(\Delta t) \\
=\left[G_{f}\left(S^{n+1}\right)-G_{c}\left(\bar{S}^{n+1}\right)\right] \Delta t+\left[G_{c}\left(\bar{S}^{n+1}\right)-G_{c}\left(\bar{S}_{h}^{n+1}\right)\right] \Delta t+o(\Delta t) .
\end{array}
$$

We note that the error consists of two parts. The first term represents the error due to artificial boundary conditions in (4.6) $\left(f_{w}=1\right.$ on $\left.\Gamma_{T}^{\mathrm{in}}\right)$ and the second term represents the error due to the incorrect value of the averaged saturation. Next, we analyze the error due to artificial boundary conditions.

First, to estimate $G_{c}\left(\bar{S}^{n+1}\right)-G_{c}\left(\bar{S}_{h}^{n+1}\right)$, note that both $I\left(\bar{S}_{h}^{n+1}\right)$ and $I\left(\bar{S}^{n+1}\right)$ are solutions of equation (4.6) at certain time instants $\tau^{n+1}$ and $\tau_{h}^{n+1}$. Indeed, letting $T=T_{i}$, we have $I\left(\bar{S}_{h}^{n+1}\right)=\chi_{i}\left(x, \tau_{h}^{n+1}\right)$ and $I\left(\bar{S}^{n+1}\right)=\chi_{i}\left(x, \tau^{n+1}\right)$, where $\tau^{n+1}$ and $\tau_{h}^{n+1}$ are determined, in absence of interpolation errors, by the constraint (4.5). Assuming that $G_{c}$ is a continuously differentiable function, we derive that

$$
G_{c}\left(\bar{S}^{n+1}\right)-G_{c}\left(\bar{S}_{h}^{n+1}\right)=G_{c}^{\prime}\left(\bar{S}_{*}^{n+1}\right)\left(\bar{S}^{n+1}-\bar{S}_{h}^{n+1}\right),
$$

for some $\bar{S}_{*}^{n+1}$ between $\bar{S}^{n+1}$ and $\bar{S}_{h}^{n+1}$. Thus, $G_{c}\left(\bar{S}^{n+1}\right)-G_{c}\left(\bar{S}_{h}^{n+1}\right)$ is bounded if $G_{c}^{\prime}$ is bounded uniformly in $(0,1)$. This is a reasonable assumption if there are no sharp fronts in $\bar{S}$. This is the case in regions where the interpolation operator is employed. For our analysis, it is sufficient to assume that

$$
\left|G_{c}\left(\bar{S}^{n+1}\right)-G_{c}\left(\bar{S}_{h}^{n+1}\right)\right| \leq A_{T}^{n+1}\left(\bar{S}^{n+1}-\bar{S}_{h}^{n+1}\right),
$$


where $A_{T}^{n+1}$ is uniformly bounded independent of $\bar{S}^{n+1}$ and $\bar{S}_{h}^{n+1}$. This holds if $G_{c}$ is a Lipschitz function. In particular, $A_{T}^{n+1}$ is bounded in regions where the interpolation operator is employed. Consequently, (6.3) becomes

$$
\delta^{n+1}-\delta^{n}=\left[G_{f}\left(S^{n+1}\right)-G_{c}\left(\bar{S}^{n+1}\right)\right] \Delta t+A_{T}^{n+1} \Delta t \delta^{n+1}+o(\Delta t) .
$$

Equivalently we have

$$
\left(I-A_{T}^{n+1} \Delta t\right) \delta^{n+1}=\delta^{n}+\left[G_{f}\left(S^{n+1}\right)-G_{c}\left(\bar{S}^{n+1}\right)\right] \Delta t+o(\Delta t) .
$$

Using (6.4), one can now show that the error contribution from $G_{c}\left(\bar{S}^{n+1}\right)-G_{c}\left(\bar{S}_{h}^{n+1}\right)$ is small provided that $1 /\left(1-A_{T}^{n+1} \Delta t\right) \leq 1+C \Delta t, C>0$, where $C$ is uniformly bounded constant. Indeed, since the initial conditions for (4.6) are the same as for the global equation, we have that $\delta^{0}=0$. Hence,

$$
\begin{aligned}
\left|\delta^{n}\right| & \leq o(\Delta t)+\Delta t \sum_{k=0}^{n-1}(1+C \Delta t)^{k}\left|G_{f}\left(S^{n-k}\right)-G_{c}\left(\bar{S}^{n-k}\right)\right| \\
& \leq o(\Delta t)+\left[\frac{e^{C(n \Delta t)}-1}{C}\right]\left[\max _{1 \leq i \leq n}\left|G_{f}\left(S^{i}\right)-G_{c}\left(\bar{S}^{i}\right)\right|\right] .
\end{aligned}
$$

Since $n \Delta t=t$, we conclude that the error contribution from $G_{c}\left(\bar{S}^{n+1}\right)-G_{c}\left(\bar{S}_{h}^{n+1}\right)$ is bounded at finite time.

Next, we analyze the error contribution from $G_{f}(S)-G_{c}(\bar{S})$. As have been mentioned already, the error $G_{f}(S)-G_{c}(\bar{S})$ is controlled via proper boundary conditions. To understand how this error behaves, we observe that in typical macroscale models based on homogenization, the function $G_{f}$ is approximated independent of boundary conditions as a product of coarse scale functions,

$$
G_{f}(S)=\overline{v \cdot \nabla f(S)} \approx v^{*} \cdot \nabla f^{*}=\frac{1}{|T|} \int_{\partial T} f^{*}(\bar{S})\left(v^{*} \cdot n\right) d s,
$$

where $v^{*}$ and $f^{*}$ denote coarse scale quantities. Here $f^{*}$ depends on $\bar{S}$, but can also depend on other coarse scale quantities, e.g., $\nabla \bar{S}$, and etc.

This type of macro-scale modeling is valid when the characteristic length scale of the transport is much smaller than the coarse mesh size, when the saturation field is smooth, and when macroscopic effects at scales comparable to, and larger than the coarse mesh size can be modeled using functions involving coarse scale quantities only. The latter is the main assumption of the approaches that use pseudo relative permeabilities. If the characteristic length scale of the transport is much smaller than the coarse mesh size, then $G_{f}(S) \approx G_{c}(\bar{S})$, and the error made in the approximation of (6.6) depends on the ratio between the characteristic length scale and the coarse block size. Indeed, in this case, it can be shown that $S$ converges to $\bar{S}$ as the characteristic length scale approaches zero. From here, one can easily obtain that $G_{f}(S)=\int v$. $\nabla f(S) \phi d x \rightarrow \int v^{*} \cdot \nabla f(\bar{S}) \phi d x$, for any smooth test function $\phi$, where $v^{*}$ is the average velocity defined as harmonic averages along streamlines. Consequently, $G_{f}(S) \approx$ $G_{c}(\bar{S})$. If the saturation field is smooth, then the error is of order of coarse mesh size. Thus, in all of the mentioned situations, the proposed algorithm should allow $G_{f}$ to be adequately modeled with $G_{c}$. One can also show that $G_{f}(S) \approx G_{c}(\bar{S})$ for transport with small diffusion (when Peclet number is of order 1 at the small scale, 
cf. [24]) using homogenization theory as well as for purely hyperbolic transport if correlation length is small using perturbation analysis.

Next, we discuss the approximation $G_{f}(S) \approx G_{c}(\bar{S})$ without assuming a scale separation. However, we assume that the evolution of two-phase flow velocity is strongly influenced by the single-phase velocity and is given by $v_{t p} \approx A^{*}(x, t) v_{s p}$, where $A^{*}$ is a scalar coarse-scale function, $v_{t p}$ and $v_{s p}$ denote two-phase and singlephase flow velocities, respectively. This assumption holds if streamlines do not change dramatically. Denote by $\tau$ time-of-flight for single-phase flow velocity, $v_{s p} \cdot \nabla \tau=1$. For simplicity, we restrict ourselves to 2-D and consider a coordinate system $(\tau, \psi)$, where $\psi$ is the streamline function defined by $\operatorname{curl}(\psi)=v_{s p}$. Then, the two-phase flow saturation equation has the form

$$
\phi \frac{\partial S}{\partial t}+A^{*}(\tau, \psi, t) f(S)_{\tau}=0
$$

where $A^{*}(\tau, \psi, t)$ denotes $A^{*}(x, t)$ in $(\tau, \psi)$ coordinate system. Assuming $A^{*}$ is a bounded smooth function, the equation (6.7) suggests that $S$ is a smooth function along the lines $\tau=$ const. Writing $G_{f}(S) \approx G_{c}(\bar{S})$ in $(\tau, \psi)$ coordinate system, we have

$$
\int_{T_{\tau, \psi}} f_{w}(S)_{\tau} d V \approx \int_{T_{\tau, \psi}} f_{w}(I(\bar{S}))_{\tau} d V
$$

where $T_{\tau, \psi}$ is an image of $T$ in $(\tau, \psi)$ coordinate system. Note that $S$ and $I(\bar{S})$ satisfy $\phi \frac{\partial S}{\partial t}+A^{*}(\tau, \psi, t) f(S)_{\tau}=0$ and $\phi \frac{\partial I(\bar{S})}{\partial t}+f(I(\bar{S}))_{\tau}=0$, respectively.

Following pseudo type approaches, a simple choice for the boundary conditions is to impose $S=1$ at the inlet of coarse blocks in Cartesian coordinate system. This type of boundary conditions overestimates the flow at the inlet of coarse blocks, especially in regions where $\tau$ varies significantly along the inlet boundary. Although our numerical simulations show that despite this error, the overall accuracy of the approach tends to be good, also for simple Cartesian grids, one can achieve higher accuracy in subgrid capturing by using curvilinear flow-based coarse blocks with boundaries given by $\tau=$ const and $\psi=$ const. Alternatively, one can use extended domains with boundaries given by $\tau=$ const and $\psi=$ const for computation of basis functions in Cartesian coarse blocks. In both of these cases, the inlet boundary condition on the coarse blocks will reflect the evolution of the saturation in the global problem, and one can show that the saturation approximation is more accurate. Thus, the use of limited global information (such as $\tau$ ) can improve the accuracy of proposed multiscale methods, in particular, they reduce the error associated with saturation distribution across the streamlines in the coarse block. However, there is still an error due to the fact that the saturation values at the inlet are not static in general. As a result, the fine-scale saturation distribution can be less steep along streamlines compared to the saturation profile obtained from the interpolation. We note that this error is not due to heterogeneities and associated with one dimensional transport. Moreover, this error is first order (in the regions away from sharp fronts) and therefore less important (see (6.5)). The reduction of this error will require nonlinear interpolation procedures (cf. (4.4) that depend on global dynamics and is a subject of future research.

We would like to mention here that in [2], we consider a more general approach that combines domain decomposition approaches with the proposed multiscale methodology. In this approach, flow conditions imposed by source terms, global boundary conditions and fine-scale features of the velocity field are allowed to change during simulations. 
6.2. Velocity approximation. In the previous section, we ignored the dynamic nature of the velocity by assuming that $v=v^{0}=v\left(x, S\left(x, t_{0}\right)\right)$ at all times $t>0$. In this section, our goal is to show that the resulting error is insignificant in the sense that it is dominated by the error caused by imposing incorrect boundary conditions on the inflow boundaries $\Gamma_{T}^{\text {in }}$. To this end, we show that the local saturation fields $I\left(\bar{S}_{i}\right)$ are approximately the same as the corresponding local saturation fields one obtains by replacing $v^{0}$ in (4.6) with the "true" velocity $v=v(x, S(x, t))$. To be more precise, we will show under some assumptions that $v$, which will be referred to as the two-phase flow velocity, can be approximated by a static part $v^{0}$, that does not depend on saturation, times a time dependent function in each coarse block.

We use homogenization theory for the analysis and ignore source terms. For brevity, we write the pressure equation (2.3) in the following compact form:

$$
\nabla \cdot(\lambda(S) K \nabla p)=0 .
$$

We assume that $K(x)=K(x, x / \epsilon)$ is a periodic function with respect to $x / \epsilon$, where $\epsilon$ is the scale of periodicity. The analysis below can be extended to random homogeneous fields, i.e. equations of the form (6.8) where $K$ is sampled from a homogeneous statistical distribution.

We start by partitioning the saturation field, $S=S_{*}+S_{n s}$, where $S_{*}$ is the smooth part of $S$ and $S_{n s}$ is a localized non-smooth part of the saturation front. In particular, $S_{n s}$ is zero in every coarse block, except the coarse blocks where the sharp front is. Then, the homogenization of (6.8) gives

$$
p=\bar{p}+N \cdot \nabla \bar{p}+\theta,
$$

where $N$ is a periodic solution of the auxiliary problem (see e.g., [9])

$$
\nabla \cdot[\lambda(S) K(\nabla N+e)]=0 .
$$

Here $e$ is the unit vector. The equation (6.10) can be understood as being formulated in a representative elementary volume. In our numerical setting, $N$ is assumed to be a periodic solution of (6.9) in a coarse block of our coarse scale partition. The latter provides a classical numerical upscaling procedure [13] and allows to relate $\bar{p}$ to the coarse scale pressure obtained using numerical upscaling methods on a coarse scale partition (see [28]). Observe that away from sharp fronts, where $S_{n s}=0$, the equation (6.10) reduces to

$$
\nabla \cdot\left[\lambda\left(S_{*}\right) K(\nabla N+e)\right]=0 .
$$

Because $S_{*}$ is smooth within the coarse block, it can be easily proved that $N$ can be approximated by a static function $\tilde{N}$, where $\tilde{N}$ is a periodic solution of

$$
\nabla \cdot[K(\nabla \tilde{N}+e)]=0 .
$$

Again, this local problem can be formulated in a coarse block. Furthermore, the solution of the pressure equation at initial time,

$$
\nabla \cdot\left[\lambda\left(S^{0}\right) K \nabla p^{0}\right]=0,
$$

allows the following multiscale expansion:

$$
p^{0}=\bar{p}^{0}+\hat{N} \cdot \nabla \bar{p}^{0}+\theta^{0},
$$


where $\hat{N}$ can be approximated by $\tilde{N}$, assuming $S^{0}$ is smooth within the coarse block. Moreover, from (6.9) and (6.11) we deduce now that the dynamic two-phase flow velocity $v$ and the initial velocity $v^{0}$ can be approximated by

$$
\begin{gathered}
v \approx v_{\epsilon}=\lambda(S) K(I+\nabla \tilde{N}) \nabla \bar{p}, \\
v^{0} \approx v_{\epsilon}^{0}=\lambda\left(S^{0}\right) K(I+\nabla \tilde{N}) \nabla \bar{p}_{0} .
\end{gathered}
$$

We need only to show that $v_{\epsilon}$ can be approximated locally (inside each $T_{i}$ ) by a constant multiple of $v_{\epsilon}^{0}$. Indeed, the interpolation operators are invariant under a (positive) scaling of the velocity. In particular, all interpolation operators that stem from a velocity field $v_{\epsilon}^{0}$ of the form $v_{\epsilon}^{0}=\alpha v_{\epsilon}$ where $\alpha$ is a positive time-dependent function are identical. This can be shown by rescaling the time variable in (4.6).

To argue that $v_{\epsilon}^{0} \approx \alpha v_{\epsilon}$ for some positive function $\alpha$, we note first that $\nabla \bar{p}$ and $\nabla \bar{p}^{0}$ can be approximated in each coarse block by constants vectors (first order approximation assuming smooth homogenized pressure profile). Moreover, $\lambda(S)=\lambda\left(S_{*}\right)$ away from sharp fronts. Thus, if $\nabla \bar{p}$ and $\nabla \bar{p}^{0}$ have approximately the same directions, then $v \approx \alpha v^{0}$ for some positive and possibly time-dependent function $\alpha$. The latter assumption holds if the coarse scale streamlines (flow paths tangential to the velocity at every point) on the coarse grid do not change significantly in the simulations. Though this may not be the case in general, it is common to have little variation in the streamline paths for two-phase flow problems with a so-called moderate mobility ratio. The mobility ratio is the ratio of the extremal values for the total mobility $\lambda(S)$. In fact, some simulation schemes assume implicitly that the streamlines vary on a slow time scale. For instance, streamline methods, which are widely used for porous media flow applications, are based on a sequential operator splitting of the coupled system (2.3)-(2.4), commonly referred to as an IMPES (Implicit Pressure Explicit Saturation) procedure. In this procedure, one solves first the pressure equation using saturation values from the previous time-step. The velocity field is then held constant when the saturation profile is advanced to the next time-step. However, a prerequisite for achieving accelerated speed with streamline simulations is that we can use large time-steps for the pressure equation. In other words, one has to keep the streamlines fixed for a certain period of time. This simplification of the two-phase flow model has been justified for problems with moderate mobility ratios, and this is a key to the success of streamline methods.

7. Numerical examples. We now use the proposed methodology to model incompressible and immiscible two-phase flow on test cases with permeability and porosity from model 2 of the $10^{\text {th }}$ SPE comparative solution project, an SPE Benchmark used to compare and validate upscaling techniques [12]. The model, which is available for download at www.spe.org/csp, consists of a Tarbert formation on top of a fluvial Upper Ness formation. Although both formations are very heterogeneous, the Upper Ness formation gives rise to more complex flow. This is due to the fact that the Upper Ness formation consists of a bundle of intertwined high permeable flow channels that carry majority of the fluid flow, whereas the porous structures in the Tarbert formation are relatively smooth in comparison. We employ here mostly data modeling parts of the fluvial Upper Ness formation. Since fluvial formations are particularly hard to upscale, the Upper Ness formation should serve as an appropriate model for testing and validation of the proposed multiscale method. The Upper Ness model is Cartesian and consists of $60 \times 220 \times 50=6.6 \cdot 10^{5}$ grid cells.

We assume that the reservoir is initially fully oil-saturated, and inject water at a constant rate in grid cells penetrated by a vertical well at the center of the domain. 
We then produce whatever reaches the producers which are vertical wells located at each of the four corners. The water and oil mobilities are defined by

$$
\lambda_{w}(S)=\frac{S^{2}}{\mu_{w}} \quad \text { and } \quad \lambda_{o}(S)=\frac{(1-S)^{2}}{\mu_{o}},
$$

where the water and oil viscosities are assumed to be equal: $\mu_{w}=\mu_{o}=0.003 \mathrm{cp}$.

To measure the overall accuracy of a saturation solution we compute the error in the fine- and coarse-grid saturation profiles relative to a reference solution,

$$
e\left(S, S_{\mathrm{ref}}, t\right)=\frac{\left\|\phi S_{\mathrm{ref}}(\cdot, t)-\phi S(\cdot, t)\right\|_{L^{2}}}{\left\|\phi S_{\mathrm{ref}}(\cdot, t)-\phi S_{\mathrm{ref}}(\cdot, 0)\right\|_{L^{2}}} .
$$

Here time is measured in pore-volumes injected (PVI), i.e., time measures the fraction of the total accessible pore-volume in $\Omega$ that has been injected into $\Omega$.

For all test cases, we use Cartesian coarse grids, and assume that the fine grid cells coincide with grid cells in original Cartesian grid. The reference solution $S_{\text {ref }}$ is computed using an implicit upstream method on the fine grid, and a corresponding coarse grid solution $S_{C}$ will be computed using the same method on a coarse grid. Moreover, note that although we use a fixed set of basis functions for MsMFEM, we solve the pressure equation repeatedly to account for mobility variations. Thus, the velocity fields in the simulations will differ from the velocity field used to generate the saturation basis functions. However, to assess the accuracy of solutions obtained using the adaptive multiscale algorithm (AMsA), we compute, at each pressure timestep, the velocity field corresponding to the reference solution for saturation, and use this velocity field in AMsA, and to compute the coarse grid solution. This allows us to monitor the error that stems from AMsA only.

7.1. Results for a two-dimensional test case. We consider first a test-case representing the bottom layer of the SPE-model. The coarse grid is defined so that each grid block contains $10 \times 10$ grid cells. The saturation plots in Figure 7.1 show that the solutions obtained using AMsA with $\alpha=0, \alpha=0.1$, and $\alpha=0.2$ (the same threshold is used in all grid blocks) are very similar to the reference solution. The solution obtained using $\alpha=1$, however, looks quite different. The sharp edges that we see in this plot are due to the fact that the boundary conditions used to generate the saturation basis functions overestimate the inflow. We therefore get too much saturation along the inflow part (with respect to the initial velocity field) of each grid block boundary. This indicates that without the adaptive component, AMsA is not able to provide plausible fine-grid saturation profiles. To achieve this, one has to build more information about the global flow problem into the saturation basis functions by specifying appropriate coarse grid blocks using global information or appropriate dynamic boundary conditions for (4.6), see [2].

Figure 7.2 shows that the accuracy of AMsA decays with increasing $\alpha$. However, for all $\alpha, \mathrm{AMsA}$ gives a significantly more accurate solution on the coarse grid than the standard upstream method on the coarse grid gives. Thus, AMsA may give enhanced accuracy on coarse grids, also without the adaptive component.

7.1.1. Computational efficiency. Except for $\alpha=1$, for which local problems are not solved during the course of a flow simulation, the computational cost of AMsA is dominated by the cost of solving the local equations (4.2). In particular, for small $\alpha$ the computational cost $C(\alpha)$ of solving (2.4) using AMsA scales roughly as

$$
C(\alpha) \sim F_{u}(\alpha) N_{t} C(0),
$$



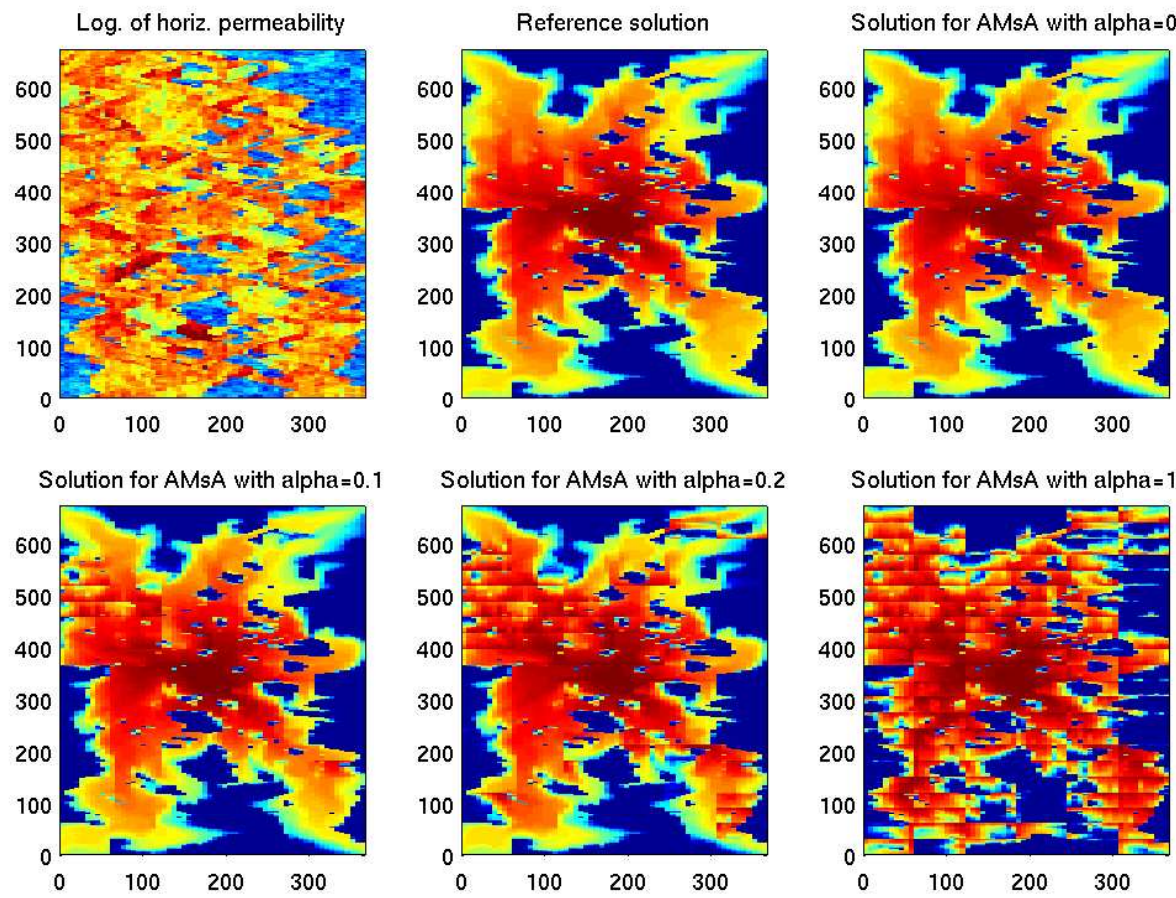

FIG. 7.1. Saturation profiles at $\sim 0.7$ PVI for simulations on the bottom layer.
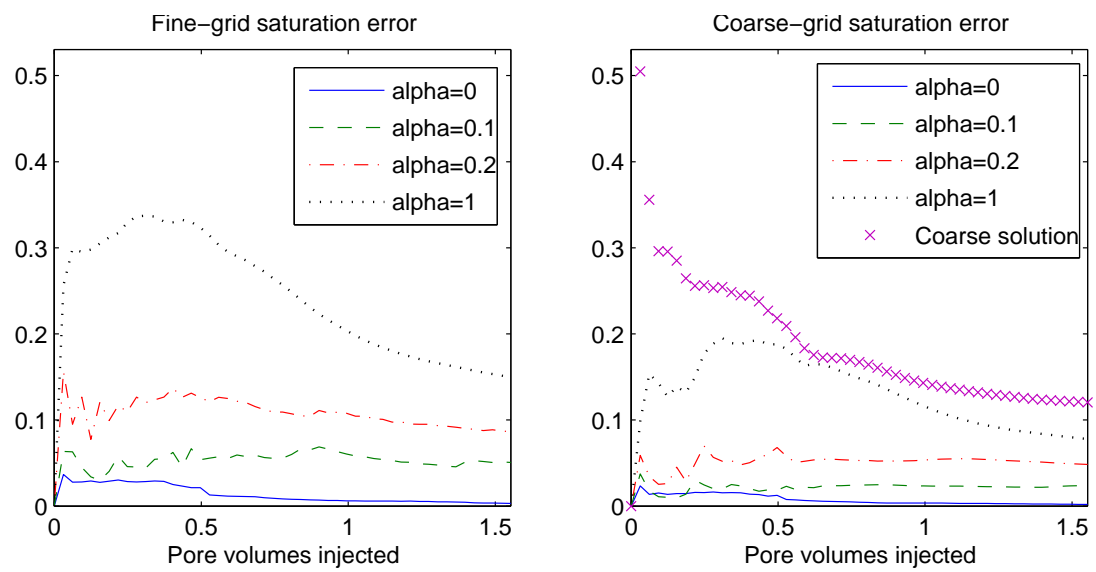

FIG. 7.2. Saturation errors for saturation solutions obtained from simulations on the bottom layer of the Upper Ness formation. The fine grid curves measure the error with $e\left(S, S_{\mathrm{ref}}, t\right)$ on the fine grid relative to the reference solution, and the coarse grid curves measure the error on a coarse grid with $e\left(\bar{S}, \bar{S}_{\text {ref }}, t\right)$ relative to the projection of the reference solution onto the coarse grid.

where $N_{t}$ is the total number of time-steps and $F_{u}(\alpha)$ is the average fraction of blocks that belong to a transient flow region.

Clearly, $F_{u}$ is a decreasing function of $\alpha$. Hence, there is a trade-off between high accuracy and low computational cost. Note also that, in addition to $\alpha, F_{u}$ depends implicitly on various factors, e.g., the coarse grid, the criteria used to identify transient 
flow regions, the fluid parameters, the heterogeneous structures, etc. In particular, AMsA is in general more efficient (and accurate) for spatially correlated variogram based permeability models than for models with fluvial heterogeneity, as is illustrated in Figure 7.3. Whereas, on average, 73 and 55 percent of the blocks in the Upper Ness model are identified to belong to transient flow regions for $\alpha=0.1$ and $\alpha=0.2$ respectively, the corresponding numbers for the Tarbert model is 46 and 27 . The potential efficiency of AMsA is therefore highly dependent on the type of model it is applied to. Relative to AMsA with $\alpha=0$, we may expect good accuracy on both coarse and fine grids, with a speed-up factor about two for models with fluvial heterogeneity, and a speed-up factor 3 or 4 for models with smoother heterogeneity.

The speed-up strongly depends on the adaptivity criteria which can be adjusted for a particular problem. In our simulations, the criteria based on gradients of the coarse-scale saturation is used. We have observed increase in speed-up when the criteria based on saturation values is used. Without the adaptive component, i.e., for $\alpha=0$, the computational complexity of AMsA is comparable to the complexity of coarse grid simulations using pseudofunctions. As we mentioned earlier, the accuracy of AMsA can be improved by choosing adaptive coarse gridding. This procedure will also enhance the efficiency of AMsA, because it localizes sharp fronts. Finally, we would like to note that the purpose of the interpolators is not primarily to get the fine scale details correct, but rather to introduce a flexible mechanism that allows us to capture the subgrid transport effects on a coarse scale.
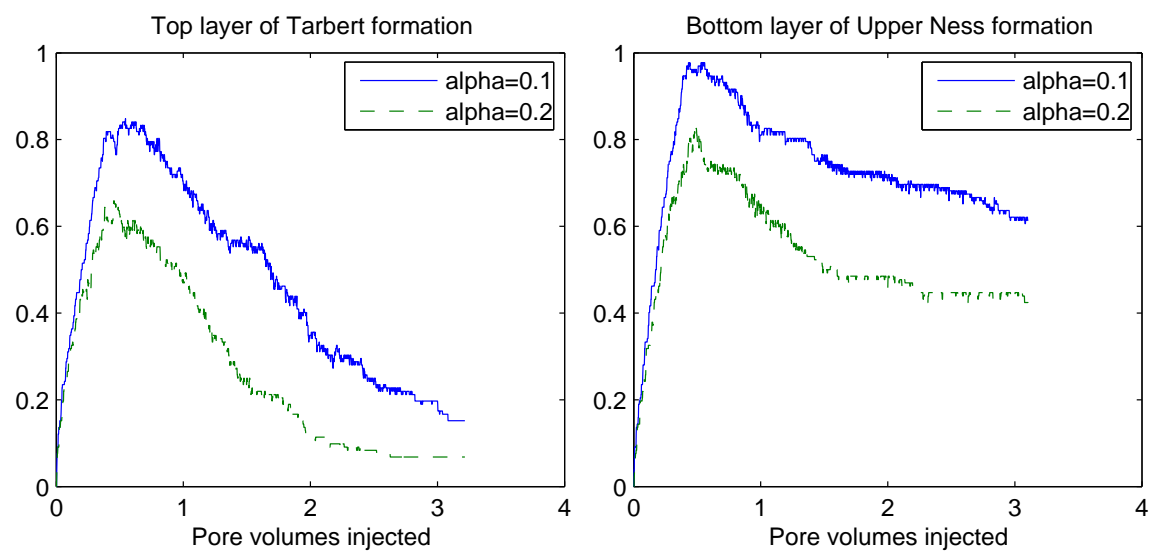

FIG. 7.3. Fraction of blocks that are identified to belong to transient flow regions during the course of two-phase flow simulations on the top layer of the Tarbert formation (left) and the bottom layer of the Upper Ness formation (right).

7.2. Three dimensional test-cases. In this section we want to examine the accuracy of AMsA when applied to two-phase flow simulations on three dimensional models from the Upper Ness formation. Here we consider only AMsA using $\alpha=0$, $\alpha=0.1$ and $\alpha=1$ in all blocks. The case $\alpha=0$ will be referred to as the domain decomposition (DD) algorithm, the case $\alpha=0.1$ will be referred to as the adaptive algorithm, and the case $\alpha=1$ will be called the multiscale algorithm.

In order for AMsA to provide a valuable tool in reservoir simulation, it should, in addition to being significantly more accurate than the coarse grid solution, capture fine-scale characteristics of the reference solution at well locations. This will be demonstrated by comparing water-cut curves (fraction of water in the produced 
fluid) for AMsA with water-cut curves for the reference solution. To get accurate production characteristics, it is essential that high flow channels are resolved adequately since high flow channels often carry majority of the flow that reach the producers. Thus, if AMsA can be used to model these regions properly, then they should provide a more robust alternative to reservoir simulation on upscaled models.

Consider first the 10 bottom layers of the Upper Ness formation, and define the coarse grid so that each grid block in the coarse grid consists of $10 \times 10 \times 5$ grid cells. Figure 7.4 and Figure 7.5 demonstrate that all AMsAs give significantly more accurate results than the solution obtained by solving the saturation equation on the coarse grid with the implicit upstream method. We notice, in particular, that the water-cut curves for the multiscale algorithm are much more accurate than the corresponding water-cut curves for the coarse grid solution. This indicates that AMsA is more capable of resolving high flow regions adequately, also without the local fine grid computations.


FIG. 7.4. Saturation errors for simulations on the bottom 10 layers of the Upper Ness formation.
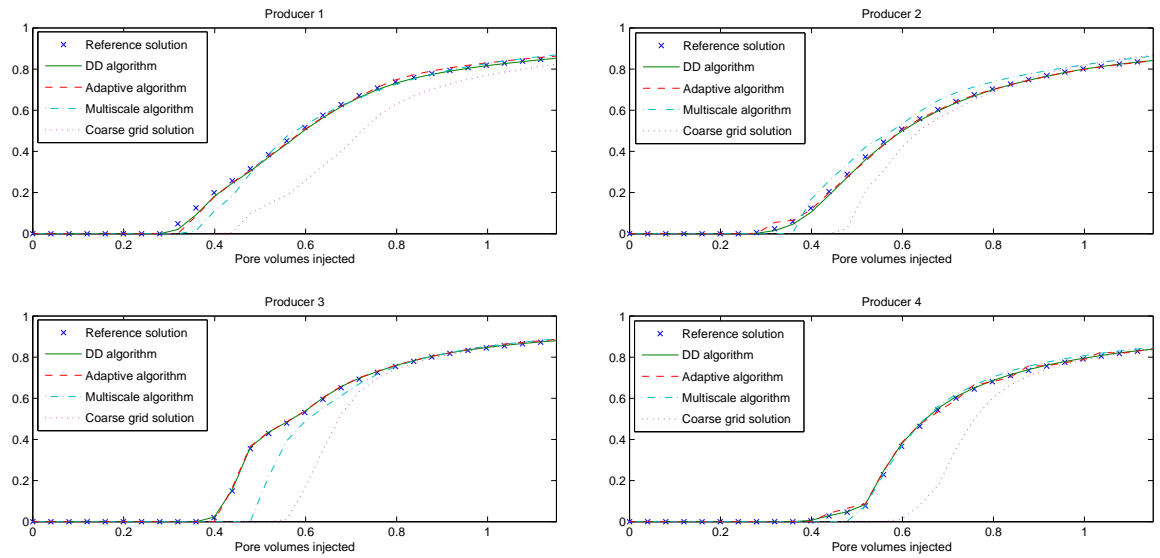

FIG. 7.5. Water-cut curves for simulations on the bottom 10 layers of the Upper Ness formation.

We turn now to the full three dimensional model of the Upper Ness formation. Since the previous examples showed that the DD algorithm seems to produce solutions that match very closely the reference solution, and it is computationally very expensive to compute a solution on the full Upper Ness model using the implicit upstream method on the fine grid, we use here the solution obtained using the DD algorithm 
as the reference solution. Again we let the coarse grid be defined so that each grid block in the grid consists of $10 \times 10 \times 5$ grid cells.

Figure 7.6 demonstrates that the errors are approximately the same as in the previous example. We observe also that the saturation error on the coarse grid for the multiscale algorithm is less than half of the corresponding error for the coarse grid solution. Furthermore, the water-cut curves for the multiscale algorithm depicted in Figure 7.7 match closely the water-cut curves for the adaptive algorithm and the DD algorithm, except possibly for producer 4 where we observe a mismatch. In contrast, the coarse grid solution continues to overestimate the breakthrough times, and thus overpredicts the oil-production. This shows that the multiscale method may be used as an alternative to pseudofunctions for enhancing accuracy of coarse grid simulations.
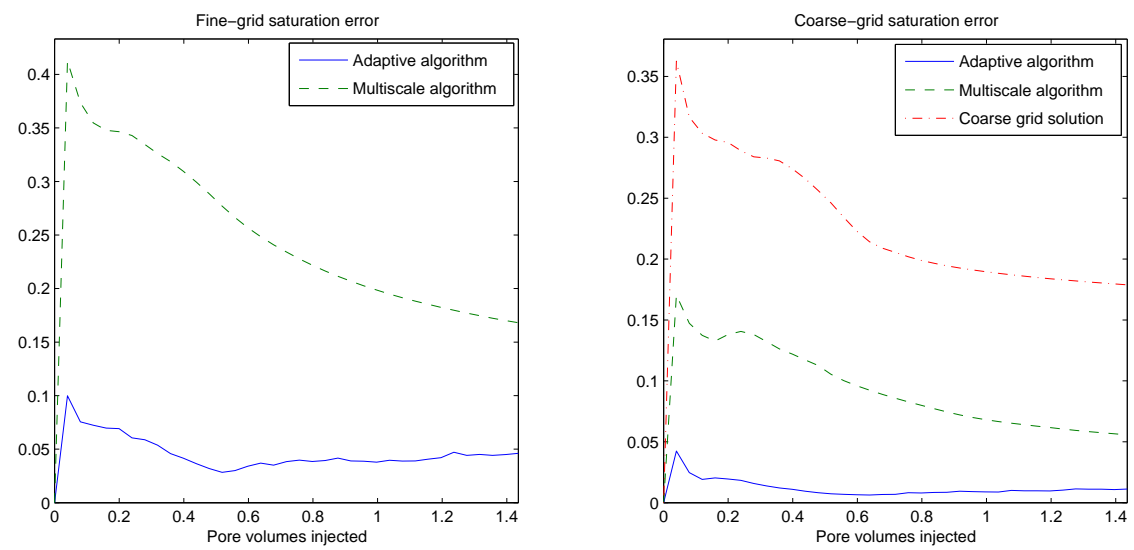

FIG. 7.6. Saturation errors for simulations on the full Upper Ness formation.


FIG. 7.7. Water-cut curves for simulations on the full model of the Upper Ness formation.

8. Concluding remarks. The main purpose of this paper has been to introduce a new (adaptive) multiscale method for solving the transport equation that arise in immiscible two-phase flow in porous media. The basic idea is to compute the global flow on a coarse grid, and map the averaged grid block saturations onto plausible saturation profiles on a finer subgrid. To enhance the accuracy of the coarse grid saturation profile, while at the same time avoid an upscaling phase involving e.g., 
the construction of pseudo relative permeability functions, we introduce a numerical scheme for solving transport equation on a coarse grid that honors fine scale structures in the velocity field in a mathematically consistent manner. Moreover, to capture rapid transitions in saturation values near propagating saturation fronts accurately, we propose to include an adaptive component in the algorithm. In the adaptive algorithm, we solve the saturation locally on a fine grid in transient flow regions.

The proposed (adaptive) multiscale method has been analyzed and tested on models with complex heterogeneous structures. It is assumed that flow velocities are given on a fine-grid scale using, e.g., a multiscale method for the elliptic part of the two-phase flow equations. In this paper a multiscale mixed finite element method has been used. This method offers high-resolution velocity fields at a low cost. However, for the enhanced resolution of velocities to give improved accuracy of flow simulations, it is necessary to account for fine scale structures in the velocity field when solving the saturation equation. The numerical results demonstrate that the multiscale method for the saturation equation is capable of providing nearly the same flow characteristics as simulations where the transport equation is solved on the scale of the underlying fine grid. The method provides therefore an efficient alternative to computing the transport on the fine grid. The analysis presented in the paper reveals and estimates the error sources and support the conclusions that we draw from the numerical results.

Acknowledgments: The authors would like to thank the referees for valuable comments and suggestions that helped improve the quality of the paper.

\section{REFERENCES}

[1] J. E. Aarnes, On the use of a mixed multiscale finite element method for greater flexibility and increased speed or improved accuracy in reservoir simulation, Multiscale Model. Simul. 2 (2004), no. 3, 421-439.

[2] J. E. Aarnes and Y. Efendiev, A multiscale method for modeling transport in porous media on unstructured corner-point grids, Proceedings of CMWR XVI, Copenhagen, Denmark, 2006 .

[3] J. E. Aarnes, V. Kippe, and K.-A. Lie, Mixed multiscale finite elements and streamline methods for reservoir simulation of large geomodels, Adv. Water Resorces. (2005), no. 28, 257-271.

[4] J. E. Aarnes, S. Krogstad, and K.-A. Lie, A hierarchical multiscale method for two-phase flow based upon mixed finite elements and nonuniform grids, To appear in Multiscale Model. Simul. (2006).

[5] T. Arbogast, Implementation of a locally conservative numerical subgrid upscaling scheme for two-phase darcy flow, Comp. Geosci. 6 (2002), 453-481.

[6] _ Analysis of a two-scale, locally conservative subgrid upscaling for elliptic problems, Siam J. Numer. Anal. 42 (2004), 576-598.

[7] I. Babuška and E. Osborn, Generalized finite element methods: Their performance and their relation to mixed methods, SIAM J. Numer. Anal. 20 (1983), 510-536.

[8] J.W. Barker and S. Thibeau, A critical review of the use of pseudorelative permeabilities for upscaling, SPE Reservoir Eng. (1997), no. 12, 138-143.

[9] A. Benesoussan, J.-L. Lions, and G. Papanicolaou, Asymptotic analysis for periodic structures, Elsevier Science Publishers, Amsterdam, 1978.

[10] Z. Chen and T. Y. Hou, A mixed multiscale finite element method for elliptic problems with oscillating coefficients, Math. Comp. 72 (2003), 541-576.

[11] M. A. Christie, Upscaling for reservoir simulation, JPT J. Pet. Tech. (1996), no. 48, 1004-1010.

[12] M. A. Christie and M. J. Blunt, Tenth SPE comparative solution project: A comparison of upscaling techniques, SPE 72469, url: www.spe.org/csp, 2001.

[13] L. J. Durlofsky, Numerical calculations of equivalent gridblock permeability tensors for heterogeneous porous media, Water Resour. Res. 27 (1991), no. 5, 699-708.

[14] Coarse scale models of two-phase flow in heterogeneous reservoirs: Volume averaged equations and their relation to existing upscaling techniques, Comp. Geosciences 2 (1998), 73-92. 
[15] W. E and B. Engquist, The heterogeneous multi-scale methods, Comm. Math. Sci. 1 (2003), $87-133$.

[16] Y. Efendiev, L. J. Durlofsky, and S. H. Lee, Modeling of subgrid effects in coarse scale simulations of transport in heterogeneous porous media, Water Resour. Res. 36 (2000), no. 8, 2031-2041.

[17] Y. Efendiev, V. Ginting, T. Y. Hou, and R. Ewing, Accurate multiscale finite element methods for two-phase flow simulations, Submitted to J. Comput. Phys.

[18] Y. Efendiev, T. Y. Hou, and V. Ginting, Multiscale finite element methods for nonlinear problems and their applications, Commun. Math. Sci. 2 (2004), no. 4, 553-589.

[19] Y. Gautier, M. J. Blunt, and M. A. Christie, Nested gridding and streamline-based simulation for fast reservoir performance prediction, Comp. Geosci. 3 (1999), 295-320.

[20] T. Y. Hou and X-H. Wu, A multiscale finite element method for elliptic problems in composite materials and porous media, J. Comput. Phys. 134 (1997), 169-189.

[21] P. Jenny, S. H. Lee, and H. A. Tchelepi, Multi-scale finite-volume method for elliptic problems in subsurface flow simulation, J. Comput. Phys. 187 (2003), 47-67.

[22] I. G. Kevrekidis, C. W. Gear, J. M. Hyman, P. G. Kevrekidis, O. Runborg, and C. Theodoropoulos, Equation-free, coarse-grained multiscale computation: enabling microscopic simulators to perform system-level analysis, Commun. Math. Sci. 1 (2003), no. 4, 715-762.

[23] J. R. Kyte and D. W. Berry, New pseudofunctions to control numerical dispersion, SPE (1975), no. 5105.

[24] J. Rubinstein and R. Mauri, Dispersion and convection in periodic porous media, SIAM J. Appl. Math. 46 (1986), 1018-1023.

[25] H. L. Stone, Rigorous black-oil pseudofunctions, SPE (1991), no. 21207.

[26] T. Strinopoulos, Upscaling of immiscible two-phase flows in an adaptive frame, Ph.D. thesis, California Institute of Technology, Pasadena, 2005.

[27] M. R. Thiele, Streamline simulation, $6^{\text {th }}$ International Forum on Reservoir Simulation, Schloss Fuschl, Austria, September 2001.

[28] X. H. Wu, Y. Efendiev, and T. Y. Hou, Analysis of upscaling absolute permeability, Discrete Contin. Dyn. Syst. Ser. B 2 (2002), no. 2, 185-204. 